\title{
Effect of vortex-core size on the flux lattice in a mesoscopic superconducting strip
}

\author{
I. Kokanović, ${ }^{1}$ A. Helzel, ${ }^{2}$ D. Babić, ${ }^{1, *}$ C. Sürgers,${ }^{3}$ and C. Strunk ${ }^{2}$ \\ ${ }^{1}$ Department of Physics, Faculty of Science, University of Zagreb, Bijenička 32, HR-10000 Zagreb, Croatia \\ ${ }^{2}$ Institute for Experimental and Applied Physics, University of Regensburg, D-93025 Regensburg, Germany \\ ${ }^{3}$ Physikalisches Institut and DFG Center for Functional Nanostructures (CFN), Universität Karlsruhe, D-76128 Karlsruhe, Germany
}

(Received 25 March 2008; published 22 May 2008)

\begin{abstract}
We present an experimental study of the vortex-motion dissipation in a mesoscopic amorphous $(a-) \mathrm{Nb}_{0.7} \mathrm{Ge}_{0.3}$ strip, with emphasis on the results for 3-8 vortex rows parallel to the long strip axis. In the isothermal voltage vs magnetic field traces, at a constant current, we observe plateaus superimposed onto a monotonic background. The plateaus appear because finite vortex-core size influences the accommodation of the flux lattice into the strip. This conclusion is drawn from a quantitative analysis, which is free of adjustable parameters, of the magnetic fields that edge the plateaus.
\end{abstract}

DOI: 10.1103/PhysRevB.77.172504 PACS number(s): 74.78.Na, 74.78.Db, 74.25.Qt, 74.25.Fy

The interest in type II superconductivity on the mesoscopic scale is nowadays growing constantly. This is a consequence of the possibility to downsize whole samples (usually thin films of thickness $d$ ), or some of their components, to dimensions comparable to lengths which govern the mixed state at a temperature $T$ and a magnetic field $B$. The most important of these are Ginzburg-Landau (GL) coherence length $\xi(T)$ and penetration depth $\lambda(T)$ as well as intervortex distance $\sim n_{\phi}^{-1 / 2}(B)$, where $n_{\phi}$ is the density of magnetic-flux quanta $\phi_{0}$. Examples of such systems are films with small perforating holes ${ }^{1,2}$ or magnetic dots, ${ }^{3}$ structured hybrids of weak-pinning and strong-pinning superconductors, ${ }^{4,5}$ singlematerial strips, ${ }^{6,7}$ etc. The miniaturization opens some questions, for instance, on the arrangement of vortices in the presence of boundary conditions to the screening currents, imposed when the in-plane penetration depth (e.g., $\lambda$ for $\lambda<d$ or $\Lambda=2 \lambda^{2} / d$, when $\lambda \gg d$ ) exceeds a lateral sample dimension. Furthermore, even $\xi$ and intervortex spacing may be of importance since approximations that consider vortices as point-like topological excitations in extended medium face limits of their applicability. To illustrate typical experimental situations, we use Refs. 6 and 7, where the GL parameters were $\xi(0) \sim 10 \mathrm{~nm}$ and $\lambda(0) \sim 1 \mu \mathrm{m}, n_{\phi}^{-1 / 2}$ $\sim 50 \mathrm{~nm}$ (at $B \sim 1 \mathrm{~T}$ ), while $d \sim 50-200 \mathrm{~nm}$ strips were $w \sim 150 \mathrm{~nm}-2 \mu \mathrm{m}$ wide.

In this Brief Report, we report on a mesoscopic phenomenon detected in the vortex transport across a weak-pinning $a-\mathrm{Nb}_{0.7} \mathrm{Ge}_{0.3}$ strip of a length $l \approx 25 w$. The voltage $(V)$ vs $B$ isotherms, at a constant applied current $I$, exhibit plateaus superimposed onto a monotonic background. The background strongly depends on $B$ and $T$, signifying a thermally activated vortex motion in the bulk. On the other hand, the plateaus display a $T$-independent regularity in $B$, suggesting an effect linked to a small number of vortex rows parallel to the long strip axis, as their center-to-center separation $b \propto n_{\phi}^{-1 / 2}$ is comparable to $w$. Plateaus can be explained by taking into account the finite size of vortex cores, i.e., their effective radius $\xi$. As $B$ increases, $b$ shrinks, and as long as $w / b$ is not an integer, this process is continuous. When $w / b$ takes an integer value, an extra row could be smoothly accommodated within the strip only if the vortices were point objects-which is not the case. Hence, there is a blockage in the increase in $n_{\phi}$, and implicitly of $V$, until $B$ grows to satisfy $(w-2 \xi) / b$ being an integer when the cores of vortices that border the sample edges can fit into the superconductor. After that, the decrease in $b$ proceeds uninterruptedly until the above condition is enforced for the next row, again resulting in a plateau, etc. We confirm this picture quantitatively-without adjustable parameters-by comparing the experimental data to a model of a regular triangular lattice with the triangle base parallel to the strip edge.

The electron-beam lithography and magnetron sputtering were combined in a single-step lift-off process to produce a strip of $w=260 \mathrm{~nm}, d=60 \mathrm{~nm}$, and $l=6.4 \mu \mathrm{m}$ referring to the center-to-center distance between the contacts for measuring $V$ (formed by perpendicularly protruding strips of the same $w$ as above). The width uniformity along the strip was sufficient for using spatially independent $w$ in addressing the results quantitatively. Furthermore, although thinner films have somewhat weaker pinning, ${ }^{2,8}$ the above $d$ provides sufficient volume for ensuring homogeneous properties of the strip at the small $w .{ }^{7}$ Measurements were carried out in a ${ }^{3} \mathrm{He}$ cryostat by a low-frequency $(29 \mathrm{~Hz})$ ac method in the fourpoint configuration. $B$ was perpendicular to the film plane and $I$ along the strip set a transversal vortex velocity $u$. The superconducting transition temperature and normal-state resistivity of the sample are $T_{c}=3.1 \mathrm{~K}$ and $\rho_{n}=3.1 \mu \Omega \mathrm{m}$, respectively. Using this and $-\left(d B_{c 2} / d T\right)_{T=T_{c}}=2.0 \mathrm{~T} / \mathrm{K}$, where $B_{c 2}$ is the upper critical magnetic field, we calculate ${ }^{7-9}$ the GL parameters: $\xi(0)=7.3 \mathrm{~nm}, \quad \lambda(0)=1.05 \mu \mathrm{m}$ $[\Lambda(0)=36.75 \mu \mathrm{m}]$, and $\kappa=\lambda(0) / 1.63 \xi(0)=88$.

The main experimental finding of this study is displayed in Figs. 1(a) and 1(b), where we plot the selected $V(B, T=$ const $)$ curves in the range $1.6 \mathrm{~K} \leq T \leq 2.6 \mathrm{~K}$ with the right-hand scale showing $V$ normalized to the normalstate voltage $V_{n}$. These results were obtained by slow $B$ sweeps $(\sim 1 \mathrm{mT} / \mathrm{s})$ at a constant $I=332 \mathrm{nA}$. Even in Fig. $1(\mathrm{a})$, one can notice plateaus in $V(B)$ at $V \ll V_{n}$, which are better seen in Fig. 1(b), where the same data are plotted for $V$ below $5 \%$ of $V_{n}$. The plateaus are superimposed onto a strongly $(B, T)$-dependent background; their positions being set by $B$ while the role of $T$ is restricted to influencing the plateau width mildly. A given plateau extends between some $B=B_{1}$ and $B=B_{2}>B_{1}$, as indicated in Fig. 1(b) by the dashed 

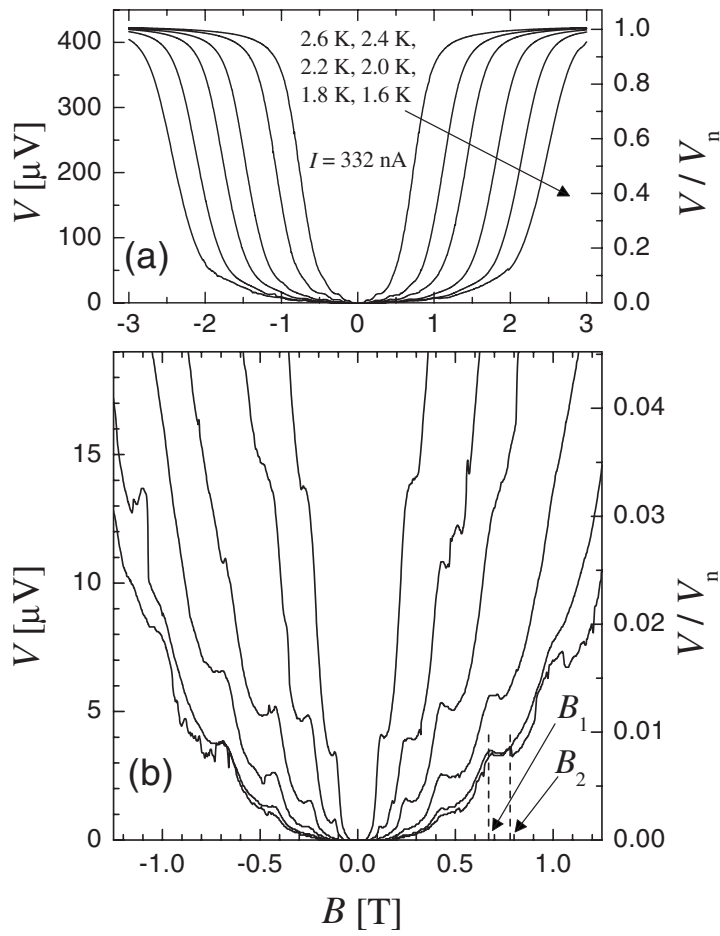

FIG. 1. (a) Selected $V(B, T=$ const $)$ at $I=332 \mathrm{nA}$, for $T$ between $1.6\left(T / T_{c} \approx 0.51\right)$ and $2.6 \mathrm{~K}\left(T / T_{c} \approx 0.83\right)$. The right-hand scale shows $V$ divided by the normal-state voltage $V_{n}$. (b) A zoom into the plot in (a), for $V / V_{n}<0.05$, revealing the plateaus more clearly. $B_{1}$ and $B_{2}$ are delineated by the dashed lines and arrows.

lines and arrows. Well-defined plateaus appear at low $B$. As $B$ increases, the plateau structure is gradually smeared out by the steeply increasing background, so, in this case, $B_{1}$ and $B_{2}$ correspond to changes in the curvature of $V(B)$. Qualitatively, the strong $(B, T)$ dependence of the background in $V$ $=\phi_{0} n_{\phi} u l$ relates to that of bulk $u$-governed by a thermally activated dynamics - as well as to the smoothness of $n_{\phi}(B)$ outside the plateau regions, while the plateaus reflect a mesoscopic effect: a retarded $n_{\phi}(B)$ response due to the finite $\xi(T)$ and small $w$. One must tune $I$ carefully to detect the above fine structure of $V(B, T) . V(I)$ is linear at small $I$ - which is favorable - but the signal-to-noise ratio is low. If $I$ is too high, the vortex cores undergo $(I, B, T)$-dependent changes due to strongly nonequilibrium phenomena, ${ }^{8}$ which results in a distortion of $V(B)$. As a compromise, we used $I$ corresponding to a current density $\sim 20 \mathrm{MA} / \mathrm{m}^{2}$, being high enough for producing a reliable signal but causing no substantial departure from linear $V(I)$ over the relevant $(B, T)$ range. ${ }^{9}$

Vortices can enter a strip of $w \ll \Lambda$ when $B$ exceeds $B_{m}=\pi \phi_{0} / 4 w^{2} \quad$ (metastable penetration) or $B_{s}=\left(2 \phi_{0} / \pi w^{2}\right) \ln (\alpha w / \xi)$ (stable configuration), where $\alpha$ is either $1 / 4$ or $2 / \pi$, depending on the details of the calculation. ${ }^{10}$ Due to the pinning force, the onset of the dissipation in our experiment takes place at $B \geq B_{m}, B_{s}{ }^{11}$ The model we use to explain $B_{1}$ and $B_{2}$ is sketched in Figs. 2(a) and 2(b). In Figs. 2(a) and 2(b), the strip is represented by the gray area while vortices are depicted by the dots (a) or solid circles (b), respectively. For brevity, we focus on the vortex arrangement that leads to the final result and implic-
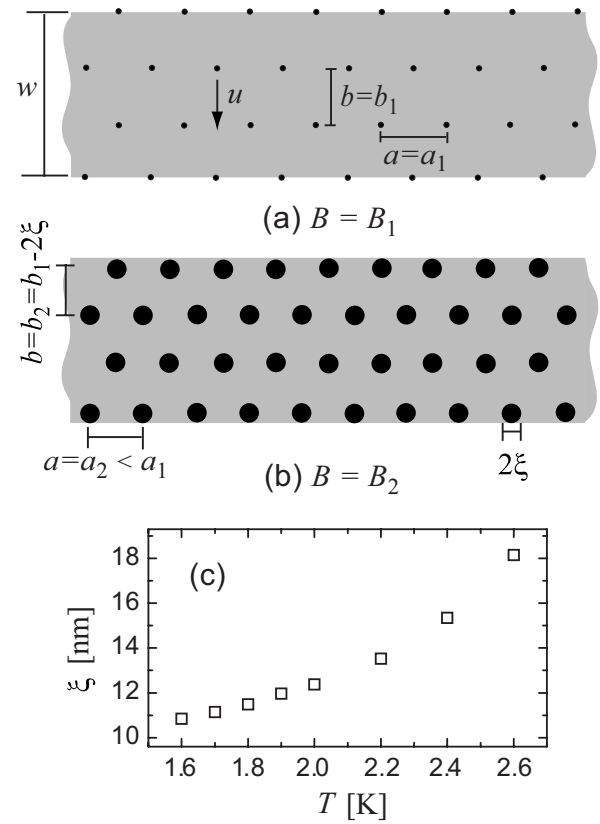

FIG. 2. Schematic representation (not to scale) of the model used in the calculation of (a) $B=B_{1}$ and (b) $B=B_{2}$. The strip is shown by the gray areas. The lattice parameter $a$ and row separation $b$ are measured from the centers of the vortex cores, the finite size of which is disregarded in (a) but taken into account in (b). The direction of $u$ is indicated in (a) and $\xi(T)$, which is found from the $B_{c 2}(T)$ curve, is plotted in (c).

itly rules out other solutions to the problem. It corresponds to an undeformed triangular lattice oriented with respect to the strip as shown. The lack of a lattice deformation follows from the large compression modulus $C_{11}$, expected theoretically, and for $a-\mathrm{Nb}_{0.7} \mathrm{Ge}_{0.3}$ also experimentally demonstrated. ${ }^{7}$ The lattice can leave a fingerprint in $V$ if the mean relative positions of vortices do not change during their transport. This holds not only when all vortices have the same $u$, i.e., in the absence of pinning, but also if the main effect of pinning causes a shear between vortices moving at different $u$, since the shear conserves the time-averaged symmetry of a lattice. As will be shown later in more detail, the shear is a viable scenario because $a-\mathrm{Nb}_{0.7} \mathrm{Ge}_{0.3}$ is a weakpinning material with a small shear-stress modulus $C_{66}{ }^{2,5}$

Let us suppose that $B$ is such that there are three rows of vortices within the strip separated by $b$ and not being close to the edges. As $B$ increases, $b$ can smoothly decrease until $w=3 b=3 b_{1}$ at some $B=B_{1}$, where four rows could accommodate within the strip if vortices were point objects. Keeping in mind the physical meaning of $B_{1}$-as a field at which the continuous shrinkage of $b$ stops for three rows-one can calculate it from the geometrical construction in Fig. 2(a). The fourth row cannot stabilize within the sample before $B=B_{2}>B_{1}$, which is depicted in Fig. 2(b) and corresponds to $w-2 \xi=3 b=3 b_{2}$. For $B_{1}<B<B_{2}, n_{\phi}$ remains constant because it could grow only by the energetically unfavorable compression of the three-row lattice along the strip. By using Figs. 2(a) and 2(b), together with the lattice parameter $a_{j}=(4 / 3)^{1 / 4}\left(\phi_{0} / B_{j}\right)^{1 / 2}=(4 / 3)^{1 / 2} b_{j}$ at $B=B_{j}$, we obtain by induction 


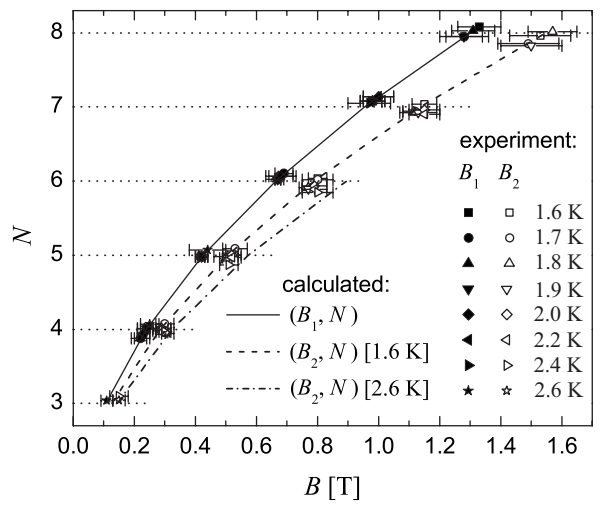

FIG. 3. $\left(B_{j}, N\right)$ obtained by inserting experimental $B_{j}$ into Eq. (1), plotted by the solid $(j=1)$ and open $(j=2)$ symbols. The solid, dashed and dashed-dotted lines connect $\left(B_{j}, N\right)$ points (as indicated) calculated by using integer values of $N$. The horizontal dotted lines are guides to the eye.

$$
B_{j}(N)=\phi_{0}\left(\frac{3}{4}\right)^{1 / 2} \frac{(N-1)^{2}}{w_{j}^{2}}, \quad j=1,2,
$$

for any number $N \geq 3$ of rows. ${ }^{12} B_{1}$ and $B_{2}$ correspond to $w_{1}=w$ and $w_{2}=w-2 \xi$, respectively. From the $B_{c 2}(T)$ curve, we can calculate $\xi(T)=\left[\phi_{0} / 2 \pi B_{c 2}(T)\right]^{1 / 2}$ with the outcome plotted in Fig. 2(c). Note that Eq. (1) contains no adjustable parameters, i.e., $B_{1}, B_{2}, w$, and $\xi$ are all experimentally accessible and $(3 / 4)^{1 / 2}$ reflects the symmetry and orientation of the lattice.

If $u$ does not change much in the range $B_{1}<B<B_{2}$, where $n_{\phi}$ is constant, $V(B)$ will exhibit a plateau rather than changes in the curvature. Plateaus are favored when $\left(B_{2}-B_{1}\right) \propto(N-1)^{2}$ is small, since the narrowness of $\left(B_{2}-B_{1}\right)$ then suppresses a variation of $u$ within. This complies with the results in Fig. 1(b), where plateaus are best defined at low $B$, i.e., small $N$. However, one can also see that plateaus are smeared out even at a relatively low $B$ if $T$ is high enough. The underlying stronger $u(B)$ dependence in this regime is primarily caused by corrections to the vortexmotion viscosity coefficient $\eta$ through terms containing $B / B_{c 2}(T)$, which is of importance when this ratio is appreciable. $^{13}$

The presented model implies that $N$, resulting from the experimental data by inverting Eq. (1), should always have integer values regardless of whether $B_{1}$ and $B_{2}$ correspond to plateaus or to changes in the curvature. This calculation leads to Fig. 3 where the solid and open symbols represent $\left(B_{1}, N\right)$ and $\left(B_{2}, N\right)$ pairs, respectively. The horizontal error bars reflect the uncertainties in $B_{1}$ and $B_{2}$, being smaller at low $B$, where well-defined plateaus enable a more accurate extraction from the experimental curves. The calculated error bars of $N$ only slightly exceed the symbol size and are omitted for clarity. The horizontal dotted lines are guides to the eye, whereas the other lines connect $\left(B_{j}, N\right)$ points determined from Eq. (1) by inserting integer values of $N$. The solid line depicts $T$-independent $\left(B_{1}, N\right)$. The upper boundary for $\left(B_{2}, N\right)$, corresponding to $T=1.6 \mathrm{~K}\left(B_{c 2}=2.8 \mathrm{~T}\right)$, is represented by the dashed line. The highest $T=2.6 \mathrm{~K}$
$\left(B_{c 2}=1.0 \mathrm{~T}\right)$ of our measurements sets the lower boundary for $\left(B_{2}, N\right)$ at low fields - where plateaus are found-which is shown by the dashed-dotted line. Clearly, from $N=3$ up to $N=8$, above which distortions in $V(B)$ cannot be detected, Eq. (1) reproduces both $B_{1}$ and $B_{2}$.

Our model is based on taking $\xi$ as the vortex-core radius but this does not mean that the vortex is simply a cylinder of the normal state. This would violate the flux-quantization condition because at $B=B_{2}$ no supercurrent could flow continuously along the strip edges. A finite superfluid density at the edges is provided by the smoothness of the radial component of the superconducting order parameter $\psi$ away from the vortex center at $r=0$, which is in an extended superconductor given by $\psi(r) \approx \psi(\infty) \tanh (r / \xi) .{ }^{14}$ The exact properties of $\psi(r)$ close to the strip edges are outside the scope of this Brief Report but the fact remains that $\xi$, determined directly from $B_{c 2}$, poses an acceptable approximation for the effective vortex-core radius.

We complete this Brief Report by a brief consideration of the vortex motion in the bulk. Since $V(I)$ does not substantially deviate from linearity for the $I$ used, as mentioned before, we can henceforth simplify the discussion by taking $V / V_{n} \approx \rho / \rho_{n}$, where $\rho$ is the mixed-state resistivity. By employing our acquired understanding ${ }^{9}$ of $\rho(B, T)$ of $a-\mathrm{Nb}_{0.7} \mathrm{Ge}_{0.3}$, we find that the background dissipation in the regime where plateaus are observed, reveals a thermally activated vortex motion. The expression appropriate for this dynamics is $\rho=\rho_{f} \exp \left(-W / k_{B} T\right), k_{B}$ being the Boltzmann constant, where $\rho_{f}=\phi_{0}^{2} n_{\phi} / \eta$ is the flux-flow resistivity given by $u=\phi_{0} I / \eta w d$, and the exponential term reflects the reduction of $u$ by a potential $W$ originating in effects of pinning (as elaborated below). When the mechanism leading to the plateaus is disregarded, $n_{\phi}$ is given by a product of $1 / a=(3 / 4)^{1 / 4}\left(B / \phi_{0}\right)^{1 / 2}$ and a discrete part $N / w$ (available from the results shown previously) accounting for the small number of rows. The Bardeen-Stephen form $\eta \approx \phi_{0} B_{c 2} / \rho_{n}$, which is a good approximation at $B \ll B_{c 2}$, as long as $T$ is not too close to $T_{c},{ }^{13}$ leads to

$$
\frac{V}{V_{n}} \approx \frac{\rho}{\rho_{n}} \approx \phi_{0}^{1 / 2}\left(\frac{3}{4}\right)^{1 / 4} \frac{N}{w} \frac{B^{1 / 2}}{B_{c 2}} \exp \left(-\frac{W}{k_{B} T}\right) .
$$

Equation (2) and the $V(B, T)$ curves can be used to extract $W(B, T)$. In Fig. 4 , we plot $W / k_{B}$ against $B$ for all the curves measured in the range where plateaus are observed. $W / k_{B}$ is rather low-of the order of $10 \mathrm{~K}$ - and decreases with increasing $B$. Over a considerable part of the plot shown, there is a trend $W \propto B^{-1 / 2}$, which is indicated by the dashed line (a guide to the eye), apart from the wiggly structure originating from using experimental $V(B, T)$ without any smoothing. The $W \propto B^{-1 / 2}$ dependence is suggestive of a shear between vortices whose velocities mismatch because of a spatially random pinning. In this picture, $W$ is representative of a washboard potential with the minima corresponding to the stable triangular lattice and the maxima to a metastable distorted structure that locally forms during the shear. ${ }^{15} W$ is then proportional to the volume occupied by vortices which move together as a unit in order to avoid the large energy cost of compression. In the simplest approximation for a strip, i.e., assuming a shear between vortex columns (perpendicular to 


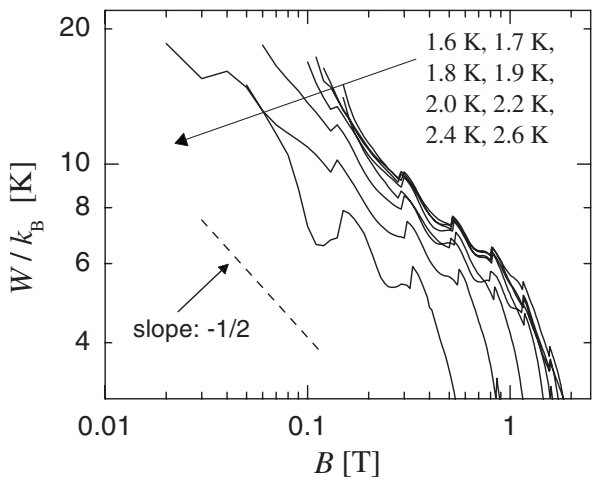

FIG. 4. Plot of $W / k_{B}$ vs $B$, calculated using Eq. (2) and experimental $V(B, T)$ without smoothing (therefore, the wiggly structure). The dashed line depicts $W \propto B^{-1 / 2}$ dependence.

the strip) of a width $a$, this volume equals $a w d$ and leads to $W \propto B^{-1 / 2}$ straightforwardly. On the other hand, the $W(T)$ dependence is weaker than that predicted in Ref. 15, where (for $\left.B \ll B_{c 2}\right)$ it follows that of the superconducting condensation energy density. This implies that $W$ contains an additional contribution of the pinning force, possibly related to the correlation units assuming the shape of crooked "rivers" of easy vortex motion, ${ }^{7}$ in average having a $T$-dependent length dif- ferent from $w$ but still remaining $\sim a$ wide. In any case, the above analysis supports previous remarks on why our results pertain to time-averaged lattice symmetry even when vortices experience a pinning landscape.

In summary, by measuring and analyzing the vortexmotion dissipation in $a-\mathrm{Nb}_{0.7} \mathrm{Ge}_{0.3}$ mesoscopic strip, we find evidence for the influence of finite vortex-core size on the flux lattice when vortices are close to the sample edges. This phenomenon is manifested in the isothermal voltage vs magnetic field curves, which exhibit plateaus superimposed onto a monotonic background. The plateaus can be explained by a simple model that contains no adjustable parameters. It combines a regular triangular lattice with the coherence length, determined independently from the upper critical magnetic field and taken as the effective vortex-core radius, which leads to remarkable agreement with the experimental data for 3-8 vortex rows within the strip. The background signal corresponds to a thermally activated vortex motion against a weak pinning potential in the bulk in a dynamics which does not disturb the lattice symmetry during vortex transport.

This work was supported by the DFG Grants No. GRK 638 and No. SFB 631, together with the MZOS Project No. 119-1191458-1008.

\footnotetext{
*Corresponding author; dbabic@phy.hr

${ }^{1}$ V. V. Moshchalkov, M. Baert, V. V. Metlushko, E. Rosseel, M. J. VanBael, K. Temst, R. Jonckheere, and Y. Bruynseraede, Phys. Rev. B 54, 7385 (1996).

${ }^{2}$ J. Bentner, D. Babić, C. Sürgers, and C. Strunk, Phys. Rev. B 70, 184516 (2004).

${ }^{3}$ J. I. Martín, M. Vélez, J. Nogués, and I. K. Schuller, Phys. Rev. Lett. 79, 1929 (1997).

${ }^{4}$ N. Kokubo, T. G. Sorop, R. Besseling, and P. H. Kes, Phys. Rev. B 73, 224514 (2006).

${ }^{5}$ D. Babić, J. Bentner, C. Sürgers, and C. Strunk, Phys. Rev. B 76, 134515 (2007).

${ }^{6}$ I. V. Grigorieva, A. K. Geim, S. V. Dubonos, K. S. Novoselov, D. Y. Vodolazov, F. M. Peeters, P. H. Kes, and M. Hesselberth, Phys. Rev. Lett. 92, 237001 (2004).

${ }^{7}$ A. Helzel, I. Kokanović, D. Babić, L. V. Litvin, F. Rohlfing, F. Otto, C. Sürgers, and C. Strunk, Phys. Rev. B 74, 220510(R) (2006).

${ }^{8}$ D. Babić, J. Bentner, C. Sürgers, and C. Strunk, Phys. Rev. B
}

69, 092510 (2004).

${ }^{9}$ For an overview of vortex-motion regimes in $a-\mathrm{Nb}_{0.7} \mathrm{Ge}_{0.3}$, see D. Babić, in New Frontiers in Superconductivity Research, edited by B. S. Martins (Nova Science, New York, 2006).

${ }^{10}$ G. Stan, S. B. Field, and J. M. Martinis, Phys. Rev. Lett. 92, 097003 (2004), and references therein.

${ }^{11}$ The equality sign applies to $T=2.6 \mathrm{~K}$ only, where the pinning is very weak and the onset of the dissipation nearly coincides with $B_{s}(\alpha=2 / \pi)=43 \mathrm{mT}$.

${ }^{12}$ Plateaus have not been found for $N<3$. Moreover, our model gives $B_{j}(N=2)$ close to $B_{m}$ and $B_{s}$, so the arrangement of vortices for $N<3$ remains an open question.

${ }^{13}$ A. I. Larkin and Yu. N. Ovchinnikov, in Nonequilibrium Superconductivity, edited by D. N. Langenberg and A. I. Larkin (North-Holland, Amsterdam, 1986).

${ }^{14}$ M. Tinkham, Introduction to Superconductivity (McGraw-Hill, New York, 1996).

${ }^{15}$ M. Tinkham, Phys. Rev. Lett. 61, 1658 (1988). 